\title{
Early surgical removal of migrated coil/stent after failed embolization of intracranial aneurysm
}

\author{
Grzegorz Turek, MD, ${ }^{1}$ Jan Kochanowicz, MD, PhD, ${ }^{2}$ Andrzej Lewszuk, MD, PhD, ${ }^{3}$ \\ Tomasz Lyson, MD, PhD, ${ }^{1}$ Justyna Zielinska-Turek, MD, ${ }^{4}$ Jan Chwiesko, ${ }^{3}$ and \\ Zenon Mariak, MD, PhD'
}

Departments of ${ }^{1}$ Neurosurgery, ${ }^{2}$ Invasive Neurology, ${ }^{3}$ Radiology, and ${ }^{4}$ Neurology, Medical University of Bialystok, Poland

\begin{abstract}
OBJECT Distal coil or stent migration is a rare, but potentially morbid complication of intracranial aneurysm embolization. At present, there is no established standard of surgical evacuation of displaced material-in particular, there is no consensus on the optimum time for such intervention. The authors report their positive experiences with an ultra-early surgical evacuation of 2 migrated coils and a flow-diverter stent.
\end{abstract}

METHODS Uncontrolled coil or stent migration occurred in $3(0.75 \%)$ of approximately 400 patients treated between 1999 and 2012 in the authors' institution. In all 3 cases, the materials moved from their intended position to the middle cerebral artery (MCA). Surgical evacuation was started immediately (within half an hour) after a futile attempt of removing them via intraarterial route, under the same anesthesia and with no active reversal of heparinization.

RESULTS No excessive bleeding was observed. Displaced coils were extracted through an incision of a branch of MCA-the anterior temporal artery, the stent was removed through a direct incision of MCA. Recombinant tissue plasminogen activator (rtPA) was injected to the stem of the internal carotid artery toward the end of the procedure, with no discernible adverse effects. Two patients were discharged with no deficit (Glasgow Outcome Scale [GOS] Score 5); the other patient was conscious with mild hemiparesis (GOS Score 4) at discharge.

CONCLUSIONS The experiences of these 3 cases suggest that immediate removal of a migrated stent/coil is feasible and may be effective. Indirect access to the MCA through its branch helps to shorten the time of temporary clipping of the artery to a minimum. Maintaining active heparinization and direct intraarterial injection of rtPA are helpful in promoting blood flow in the MCA.

http://thejns.org/doi/abs/10.3171/2015.1.JNS132788

KEY WORDS cerebral aneurysm; aneurysm embolization; coil migration; surgical evacuation; vascular disorders

$\mathrm{E}$ NDOVASCULAR coiling has been increasingly considered a "method of choice" for many patients with intracranial aneurysms. Nevertheless, the rate of complications for these procedures is estimated at $10 \%-$ $19 \%, 7,7,17,23$ a figure that continues to be high in spite of ongoing technical advances and the increasing expertise of neurovascular teams. Among the complications, intraprocedural aneurysm rupture is the most frequent $(2.9 \%-$ $8.8 \%$ ), followed by intraoperative vasospasm and thromboembolism $(2.5 \%-28 \%))^{2,16,18,19,22}$ Uncontrolled migration of coils or stents out of their destination occurs in $2 \%-6 \%$ of procedures, and in some instances these materials move out of reach of the endovascular instruments. ${ }^{1,5,7,8,13,18,19} \mathrm{In}$ such circumstances, the problem can be solved only by direct removal of the material; however, the optimum time for this kind of neurosurgical intervention remains undefined. Removal of migrated material from a major cerebral artery is a real challenge, even for an experienced vascular neurosurgeon. There is no commonly accepted standard surgical method for safe and effective extraction of displaced material from cerebral arteries..$^{13,14,21}$ Therefore, every case must be resolved on an individual basis.

ABBREVIATIONS ATA = anterior temporal artery; GOS = Glasgow Outcome Scale; ICA = internal carotid artery; ICU = intensive care unit; MCA = middle cerebral artery; $\mathrm{mRS}=$ modified Rankin Scale; $\mathrm{PCoA}=$ posterior commuicating artery; rtPA = recombinant tissue plasminogen activator .

SUBMITTED December 16, 2013. ACCEPTED January 28, 2015.

INCLUDE WHEN CITING Published online July 31, 2015; DOI:10.3171/2015.1.JNS132788.

DISCLOSURE The authors report no conflict of interest concerning the materials or methods used in this study or the findings specified in this paper. 
According to available data, the time from failed aneurysm embolization to neurosurgical intervention is specified as several hours to a few days; however, no author has concentrated on the significance of ultra-early intervention, i.e., undertaken during the same anesthesia. ${ }^{20,21}$ In this paper we share the experience we gained while dealing with 3 such complications, which occurred among nearly 400 procedures of intravascular embolization of cerebral aneurysms. In all 3 cases, direct removal of the displaced embolization material was undertaken immediately-that is, within half an hour after an unsuccessful attempt of removing material via the endovascular route.

\section{Methods}

This study was approved by the bioethics committee of the Medical University of Bialystok.

Between 1999 and 2012, approximately 400 patients diagnosed with cerebral aneurysm were treated with coil embolization at our institution. Uncontrolled intravascular migration of a coil or stent from its attempted destination to the peripheral segment of the vessel occurred in 3 cases, which corresponded to $0.75 \%$ prevalence of this kind of complication. All 3 patients were undergoing a procedure for treatment of an unruptured aneurysm and were fully conscious during the attempted embolization. Their clinical status was assessed at 3-6 months postsurgery using the Glasgow Outcome Scale (GOS) and modified Rankin Scale (mRS) during the scheduled follow-up visit.

Endovascular procedures were performed under general anesthesia with propofol, midazolam, and fentanyl. Standard dual antiplatelet therapy (aspirin $75 \mathrm{mg}$ and clopidogrel $75 \mathrm{mg}$ daily) was administered for 5 days before the procedure, and a single dose of 5000 units of heparin was administered directly before embolization. Activated clotting time was maintained at 1.5 to 2 times the baseline level throughout the procedure. ${ }^{10}$ Directly after the procedure, the standard dual antiplatelet therapy was replaced with 0.6-ml injections of low-molecular-weight heparin, administered twice a day up to 30 days after the discharge.

The Siemens Artis biplane angiographic unit was used for intravascular procedures in all patients. All procedures were performed through catheterization of the femoral artery. Mechanically detachable coils (MDS, Balt) were introduced through the leading catheter, Casasco $6 \mathrm{~F}$ (Balt), or through the microcatheter, Vasco $10+$ (Balt), in the case of 2 patients. We attempted to place a flow diverter stent-the Pipeline embolization device (ev3) in the internal carotid artery (ICA) through the Casasco $6 \mathrm{~F}$ leading catheter and Marksman microcatheter (ev3) in the case of 1 patient.

In all 3 described cases, the material moved from its intended placement to the middle cerebral artery (MCA), and eventually had to be removed via a transcranial approach. All transcranial procedures were performed by the same surgeon (Z.M.). Multiple attempts at retrieving the coil or stent via an endovascular route were undertaken before the final decision to perform a craniotomy was made. In 2 patients with displaced coils, we initially tried to pass the area of occlusion with microwires that were manually twisted in a shape of a pigtail (Synchro-10,
Stryker; Hybrid 1214D, Balt). After failure of these attempts, a microsnare device (Lasso, Balt) and then the Alligator retrieval device (ev3) were used unsuccessfully. Detailed information on endovascular handling of a partially uncurled stent is included in the report of Case 3.

In Case 3, the stent (Pipeline embolization device) uncurled only in its proximal part, while its distal end remained not only curled but also twisted. Perhaps, this was the reason why the attempts at expanding it with the aid of angioplasty proved unsuccessful, despite introducing the balloon (Scepter C, Microvention) into the proximal, uncurled part of the stent. We tried to remove the stent with a microsnare device (Lasso, Balt) and then with an Alligator retrieval device (ev3), but neither of the attempts proved successful. In actuality, we were unwilling to exert more than mild traction on these devices for fear that the vessel could be injured by the open end of the Pipeline device. Another potential risk was decoupling of the capture coil of the Pipeline device and leaving it in the vessel.

\section{Results \\ Case 1}

A 54-year-old woman presented with chronic headache, right side ptosis, and right gaze diplopia. MR angiography showed a $16 \times 12-$ mm unruptured ICA aneurysm placed in the cavernous sinus $\left(\mathrm{C}_{4}\right.$ segment of the ICA). In the first attempt at intravascular embolization, the aneurysm was completely packed with 8 spirals without sequelae complications. Follow-up angiography performed 2 months after the procedure revealed an incomplete embolization. An attempt to place an additional coil ended up in its displacement to the trunk of the MCA (Fig. 1A) with barely traceable flow in this artery. Attempts at evacuation of the coil via an endovascular route proved unsuccessful, and the patient (under continued general anesthesia) was immediately transferred to the operating theater, where a small fronto-temporal craniotomy was performed and the sylvian fissure was opened to expose the MCA. The distended peripheral $\mathrm{M}_{1}$ segment was visualized under high magnification, with the coil visible through the extremely thinned arterial walls (Fig. 2A). Consequently, the initial plan of making an incision in the trunk of the artery was abandoned out of fear of uncontrolled tearing of its wall during evacuation of the coil. A small $(\sim 1-\mathrm{mm}$ caliber $)$ branch of the $M_{1}$ was instead identified (the anterior temporal artery [ATA] feeding the anterior temporal lobe, Fig. $2 \mathrm{~A}$ and $\mathrm{B}$ ) and incised with fine microscissors. The ATA was incised only halfway through its circumference in order to prevent its contraction and to keep the incision easily accessible. Temporary clips were placed at the trunk of the MCA proximal to the coil and distally at the lower main branch of the MCA, behind the cut artery. A fine microhook was fashioned by bending the tip of an intravenous needle and used to anchor the coil, which was then successfully evacuated with the aid of minute, ultrathin microforceps (Fig. 2C). The proximal clip was released before closing the arterial incision to release the blood with possible clots. The incised ATA was closed with a mini Aesculap clip (Fig. 2D), then the peripheral clip was released, and afterward $1 \mathrm{ml}$ of rtPA solution was injected 

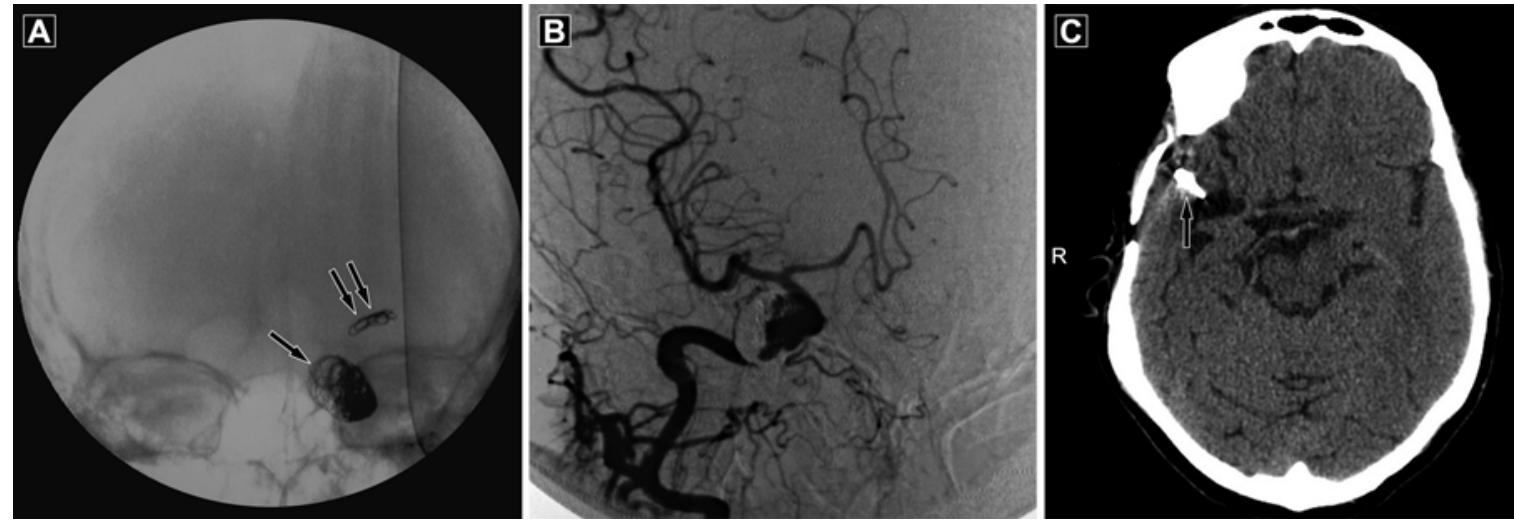

FIG. 1. Case 1. A: Radiograph obtained intraoperatively with C-arm showing a coil displaced from a cavernous sinus aneurysm (arrow) into the main trunk of the MCA (double arrows). B: Cerebral angiogram obtained 6 months after craniotomy demonstrating patent vasculature with good distal flow. C: Unenhanced CT scan of the head obtained 6 months after craniotomy revealing a modest hypodense zone in the anterior part of the temporal lobe and localization of a clip on the ATA (arrow).

into the stem of the ICA. After the injection, a persistent oozing of blood from the operating field was noted, but it ceased after 15 minutes of continuous rinsing with saline. The restoration of flow within the trunk of the MCA was confirmed with intraoperative Doppler ultrasound. The patient was transferred directly from the operating theater to the neurosurgical intensive care unit (ICU), where she was put under a "prophylactic" coma with propofol. After withdrawal of propofol, the patient had transient left hemiparesis, which resolved gradually within a few days. At discharge she was fully conscious, neurologically intact, and scored 5 on the GOS and 1 on the mRS. A followup cerebral angiogram obtained 6 months after the craniotomy revealed patent vasculature with satisfactory distal flow (Fig. 1B). A follow-up CT scan revealed a hypodense zone, approximately $2 \mathrm{~cm}$ in diameter, in the anterior part of the temporal lobe (Fig. 1C).

\section{Case 2}

A 58-year-old woman experienced subarachnoid hemorrhage and underwent clipping of an ICA aneurysm in 1983. Follow-up angiography was performed in 2008 because the patient was complaining of recurrent headaches. The examination revealed the dome of a "daughter" aneurysm, which had formed between the clip and the parent vessel. The patient decided to have this residual aneurysm treated and insisted on intravascular embolization. A coil was placed in the aneurysm, but immediately
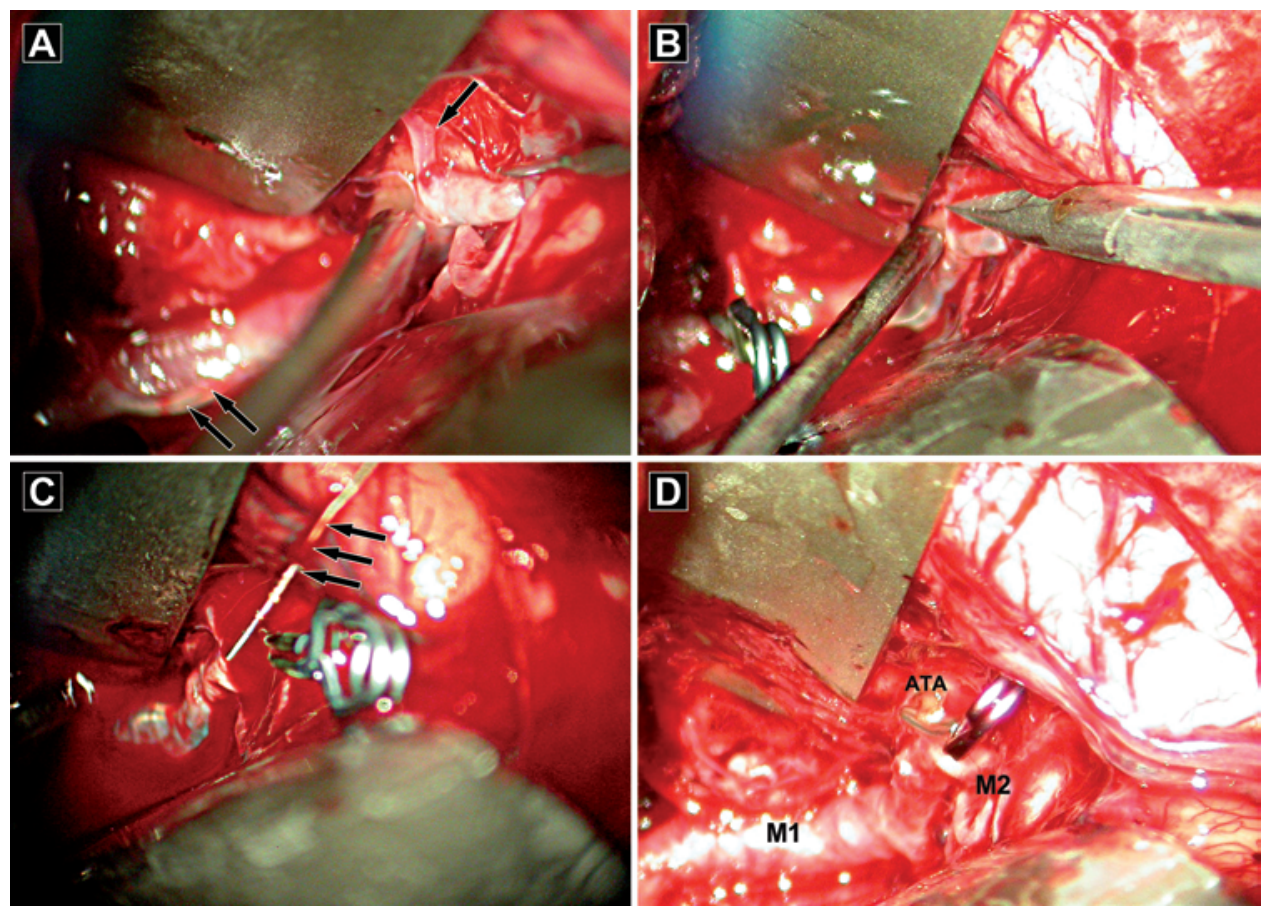

FIG. 2. Case 1. Intraoperative view of the opened sylvian fissure. A: Image obtained with focus on the ATA branching off the distal $\mathrm{M}_{1}$ segment of MCA (arrow). The coil is visible through the thinned wall of the distended MCA (double arrows). B: ATA being incised with microscissors. C: Coil being pulled out of the MCA through the incised ATA (arrows). D: ATA sealed with a clip. 
after release it moved to the trunk of the MCA, and all attempts at evacuation via an endovascular route proved unsuccessful. Absence of blood flow in the MCA was confirmed with contrast injection (Fig. 3 left). The patient was immediately transferred to the operating theater under continuous anesthesia. The former pterional craniotomy was reconstructed, and the sylvian fissure was opened. The next steps corresponded to the procedure described in Case 1, and the coil was successfully extracted through an incision of the ATA. However, in contrast to the former case, no outflow of blood was obtained from the MCA after releasing the clips. Therefore, a 2-mm incision was made in the MCA, close to its branching off from the ICA, and a ball-tipped microprobe was introduced into the artery in both directions-peripheral and proximal-to a depth of several centimeters. The artery was stretched to its sides with gentle rotating movements, which yielded a slow outflow of blood. The incision was sutured with $10-0$ intermittent stitches, and $1 \mathrm{ml}$ of rtPA solution was injected into the stem of the ICA (with subsequent oozing of blood from the operating field, easily controlled after rinsing with saline). During this time, the ICA aneurysm was prepared and secured. The trunk and the branches of the MCA were checked with intraoperative Doppler ultrasonography to confirm a stable, although low-amplitude, flow. The patient was transferred directly from the operating theater to the neurosurgical ICU, where she was put under "prophylactic" coma with propofol. After recovery from the coma, she was conscious and presented with moderate hemiparesis. When she was discharged, on the 14th day after the procedure, she was able to walk (with mild hemiparesis), live independently, and scored 4 and 2 on GOS and mRS, respectively. A follow-up CT examination revealed a significant hypodense zone in the posterior part of the frontal lobe and anterior part of the temporal lobe (Fig. 3 right). Unfortunately, the patient did not present for the follow-up angiographic examination that was scheduled for 6 months after the discharge.

\section{Case 3}

A 39-year-old man presented with migraine attacks and symptoms of atherosclerosis. Cerebral angiography revealed a right-side fusiform aneurysm involving the $\mathrm{C}_{4}-$ $\mathrm{C}_{7}$ segments of the ICA and a proximal part of the $\mathrm{M}_{1}$ segment of the MCA. Notably, neither the $A_{1}$ segment nor the posterior communicating artery $(\mathrm{PCoA})$ were visualized after injection of contrast to the right ICA (Fig. 4C). After discussing the available options with the patient, a decision was made to place a flow-diverter stent, matched to the length of the aneurysm, via an intravascular route. When placed, the stent uncurled only partially in its proximal end, and all attempts at uncurling it with an inflated balloon and extracting it with intravascular manipulators (Lasso retrieval microcatheter, Alligator retrieval device) not only proved unsuccessful but pushed the stent even more peripherally, up to the proximal part of the $\mathrm{M}_{1}$ section.

After injecting contrast medium into the right ICA, we observed persistent albeit severely restricted blood flow in the MCA (Fig. 4A). After the contrast agent was injected into the right vertebral artery, no flow in the PCoA was
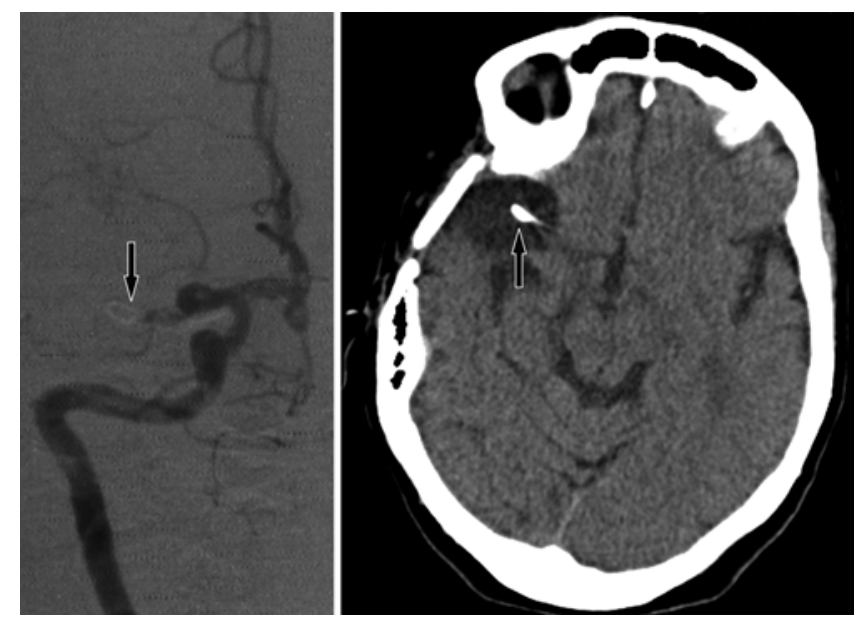

FIG. 3. Case 2. Left: Cerebral angiogram demonstrating total occlusion of the MCA by the coil displaced to the proximal $\mathrm{M}_{1}$ segment (arrow). Right: Postoperative CT image obtained 6 months after craniotomy revealing a hypodense zone in the anterior part of the temporal lobe and clip on the ATA (arrow).

documented but peripheral branches of MCA were shown to be supplied with a slow influx from the posterior temporal artery. Therefore, facing an anatomical variant of the ICA devoid of both communicating arteries and having estimated the peripheral anastomosis to be insufficient, a decision was made to remove the material by an incision of the vessel. The patient was moved to the operating theater under continuous anesthesia. Pterional craniotomy was performed and the sylvian fissure was opened according to standard practice. It was determined that a tortuous, distended intracranial portion of the ICA continued directly on to the proximal part of the $\mathrm{M}_{1}$, with no visible trace of the $A_{1}$ segment and with an aplastic PCoA. No shape or exact position of the stent within the artery was discernible by visual examination, nor by palpation with instruments. Thus a 4-mm incision of the $M_{1}$ segment was performed with no proximal clipping of the artery. With blood outflow relatively easily controlled by suction, the stent was placed with a ball-tip microprobe. This proved more difficult than anticipated, because the unfolded stent was partially buried within the intimal folds of the aneurysmal wall. Eventually, the network of the stent was identified and bent, which allowed its gradual removal throughout the incision (Fig. 4B). The incision was closed using 10-0 single sutures, the wall of the aneurysm was reinforced with TachoSil (Takeda), and $1 \mathrm{ml}$ of rtPA solution was injected into the stem of the ICA. Pulsating blood flow in the ICA and MCA was confirmed with intraoperative Doppler ultrasonography. After the procedure, the patient was transferred directly to the neurosurgical ICU where propfol-induced coma was substituted for general anesthesia. After propofol withdrawal, the patient was awake, with moderate right hemiparesis, which gradually faded away within days. At discharge, the patient was neurologically intact and scored 5 on the GOS and 0 on the $\mathrm{mRS}$. Cerebral angiography performed 6 months after the craniotomy revealed the presence of normal blood flow in the MCA (Fig. 4C). A follow-up CT examination showed 

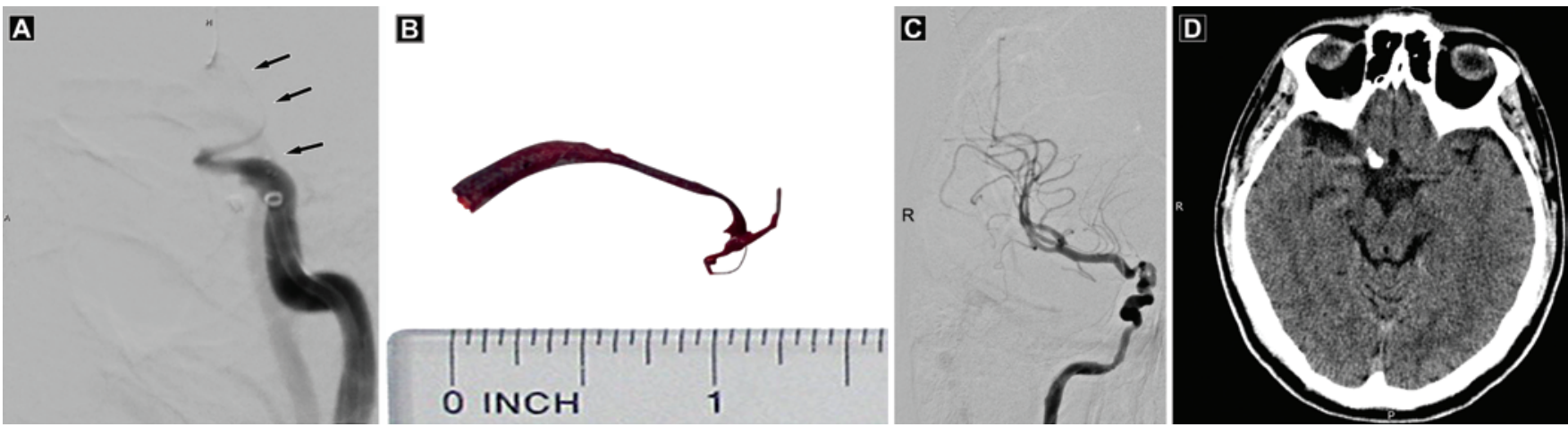

FIG. 4. Case 3. A: Cerebral angiogram showing the partially uncurled flow diverter stent (arrows) extending from the ICA into the proximal part of the MCA. Blood flow in the artery is severely restricted. The $A_{1}$ segment of the ACA is aplastic. B: Photograph of partially uncurled stent removed from the artery. C: Cerebral angiogram at 6 months after craniotomy revealing restored blood flow in the MCA. D: Unenhanced CT scan obtained 6 months after craniotomy revealing a hypodense zone in the anterior part of the temporal lobe.

a hypodense zone, about $2 \mathrm{~cm}$ in diameter, in the anterior part of the temporal lobe (Fig. 4D).

\section{Discussion}

The scarce literature regarding the complications after endovascular embolization of cerebral aneurysms is dominated by reports of partial coil stretching from the aneurysm to the lumen of the parent artery or protrusion outside of the aneurysm. In such cases, the coil can be extracted through the dome of the aneurysm; this procedure can prove troublesome, but generally remains within the skills of the vascular neurosurgeon..$^{20,21}$ Endovascular or even conservative treatment options are often also considered in such situations. ${ }^{4,5,13,14,15}$

Migration of a coil or undeveloped stent to a distant MCA is a rare condition. Only 4 reports of such complications have been published thus far, among them 1 case report from our center; this was the first published case in which a distally migrated coil was successfully evacuated from the MCA with the aid of the technique presented in the current paper. ${ }^{16}$ Due to the unique character of this complication, no routine methods of surgical evacuation of displaced material have been developed to date. A number of devices, including microsnares, microforceps, and loops, have been used to retrieve migrated material via an endovascular route. ${ }^{3,5}$ After several futile efforts, Deshmukh et al. ${ }^{3}$ reversed heparinization with protamine, performed a vast orbitozygomatic craniotomy, opened the $\mathrm{M}_{3}$ segment of the MCA, and removed a coil. Three months after the procedure, the patient presented neurologically intact. ${ }^{3}$ Heuer et al. ${ }^{9}$ reported a unique case involving a 2-year-old girl in whom a Gianturco steel coil was lost during handling of a Blalock-Taussig shunt; the coil was eventually found in the $\mathrm{M}_{1}$ segment of the MCA. A frontal craniotomy was performed 2 hours after the occlusion, and the material was removed by means of direct arteriotomy. The authors did not report on heparin reversal. After the procedure, the artery turned out to be patent, and the patient had mild hemiparesis, which resolved within 48 hours. ${ }^{9}$ Kim et al. ${ }^{11}$ reported on a series of 6 patients who experienced a parent artery occlusion fol- lowing endovascular aneurysm handling, including only 1 individual with distant coil migration to the MCA. An attempt at extracting the coil with a snare catheter proved unsuccessful, and the coil was pushed from the $\mathrm{M}_{1}$ segment to the $\mathrm{M}_{2}$ segment of the MCA. After reversal of heparinization with protamine, a pterional craniotomy was performed 2 hours following the arterial occlusion, and the coil was extracted via a small arteriotomy. After the thrombus was washing away, urokinase $(20,000 \mathrm{IU})$ was applied proximal to the arteriotomy site due to "inadequately decreased blood flow velocity." According to the authors, the patient made "an excellent recovery." 11

In our 2 patients with a migrated coil (Cases 1 and 2), we managed to remove the material without MCA arteriotomy. The surgical technique was described in detail above, in the report of Case 1. The incised, but not completely cut, ATA remained in its anatomical position and could be easily clipped (with the aid of gentle traction on its peripheral segment) immediately after the coil was evacuated through its incision and the MCA was rinsed with saline. With no necessity of incising and stitching the MCA stem, temporary clipping of the artery took no longer than 5 minutes in each of these cases. No neurological or psychological sequelae could be discerned after sacrificing the ATA, which normally supplies the anterior temporal lobe. Unfortunately, this method could not be used for the migrated and partially unfolded stent due to its size and relative stiffness. Instead, the stent was grabbed in the middle, bent, and evacuated through a 4-mm direct arteriotomy, with no temporary clipping of the MCA.

Deshmukh et al. ${ }^{3}$ and other authors recommend administration of barbiturate and mannitol until the surgical intervention. Instead, we continued general anesthesia and then prolonged it in the form of pharmacologically induced (propofol) coma for several days after the procedure. However, such a protocol can be used only when the intervention is undertaken immediately after coil migration is discerned.

In contrast to Deshmukh et al. ${ }^{3}$ and Kim et al., ${ }^{11}$ we did not use protamine, assuming that the biological halflife of heparin is relatively short ( 2 hours). Instead we ceased heparin infusion prior to transferring our patients 
from the intravascular unit to the operating theater. Noticeably, we did not observe any excessive bleeding, either during craniotomy or while handling the brain and the arteries. Moreover, the very light blood outflow from the fenestrated MCA was considered to be a problem (as in Case 2), forcing us to resort to mechanical dilation of the artery supported with an injection of rtPA into the stem of the ICA. It is noteworthy that this intraarterial injection did not produce any important problems with hemostasis, apart from temporary oozing from the exposed brain and surgical wound, which could be controlled with continuous rinsing of the operating field. This suggests that the procoagulative effect of intravascular intervention is strong and durable and that its prevention (by allowing heparin to act and promotion of fibrinolysis with topical rtPA injection) seems a prerequisite for successful and uneventful surgical removal of displaced intravascular coils.

None of the previously mentioned authors define an optimum time for surgical intervention. Deshmukh et al., ${ }^{3}$ Kim et al., ${ }^{11}$ and Heuer et al. ${ }^{9}$ performed their interventions immediately, with satisfactory outcomes. Other authors implemented intervention between a few hours and a few days following coil protrusion into the parent artery. ${ }^{20,21}$ Also our herein-presented findings seem to support the benefits of an ultra-early approach, because all our patients recovered to live independently.

One may claim that the patients would recover also if the displaced stent or coils were left in place. As no controlled study addressing the problem in question has been conducted to date (and is not likely to be feasible), one can only speculate that the degree of the MCA obturation by a displaced coil or stent is a critical factor. The flow in the MCA in our 2 patients (Cases 1 and 3) was "barely traceable," and satisfactory recovery was documented in both of these cases. In contrast, no discernible blood flow in the MCA was observed in Case 2; this patient ended up with a permanent mild hemiparesis, and more extensive hypodense area was observed on follow-up CT imaging of the brain, when compared with the other 2 patients. Undoubtedly, patients with complete MCA occlusion require immediate intervention. In contrast, angiographic evidence of even "a trace of flow" leaves a margin for other decisions (e.g., scheduled surgery or even conservative treatment).

$\mathrm{Kim}^{12}$ reported on the successful conservative treatment of a patient with suboptimal stent deployment; however, the individual presented with normal blood flow on postoperative angiography. Based on an analysis of 9 cases, Fiorella et al. ${ }^{6}$ concluded that symptomatic patients with delayed in-stent stenosis should undergo endovascular angioplasty or bypass surgery, whereas a "strategy of watchful waiting may be effective" in the case of asymptomatic subjects. However, analysis of the available angiograms from their 2 patients who qualified for angioplasty and/or bypass surgery suggests that they presented with apparently more intensive blood flow in the MCAs than our patient with insufficient stent expansion (Case 3). It is noteworthy that our case was atypical, as the patient presented with severe insufficiency of the circle of Willis, namely lack of the $A_{1}$ segment and hypoplastic PCoAs. Due to extremely weak inflow to the MCA via a cortical anastomosis with the vertebrobasilar arteries, we had virtually no other choices than radical surgical intervention.

Our experience with 3 cases is not sufficient to allow for any conclusions regarding the superiority of early surgical treatment over more conservative approaches. We can only suggest that this treatment option is feasible and may prove to be effective in patients with no flow or only weak blood flow in the MCA, in whom it is hard to predict how long vital brain functions can be maintained. Finally, legal aspects of a decision made in such a critical situation should be considered as an argument for a more aggressive approach, such as early surgical intervention.

\section{Conclusions}

Migration of a coil or stent to the distal MCA is a dangerous but potentially treatable complication of aneurysm embolization. Ultra-early neurosurgical intervention under continued general anesthesia-with simultaneous antithrombotic therapy - is a feasible and potentially effective treatment option if no blood flow or only weak flow is present in the MCA. Surgical technique with accessing the MCA via a partially cut branch (ATA) proved to be a safe and effective mode of coil evacuation, allowing the time of MCA handling to be made as short as possible and producing no discernible consequences in 2 patients treated in this way. Cooperation between vascular neurosurgeons, endovascular specialists, and neuroanesthesiologists can result in prompt and successful management of this rare but potentially morbid endovascular complication.

\section{References}

1. Casasco AE, Aymard A, Gobin YP, Houdart E, Rogopoulos A, George B, et al: Selective endovascular treatment of 71 intracranial aneurysms with platinum coils. J Neurosurg 79:3-10, 1993

2. Deng J, Zhao Z, Gao G: Periprocedural complications associated with endovascular embolisation of intracranial ruptured aneurysms with matrix coils. Singapore Med J 48:429-433, 2007

3. Deshmukh VR, Klopfenstein J, Albuquerque FC, Kim LJ, Spetzler RF: Surgical management of distal coil migration and arterial perforation after attempted coil embolization of a ruptured ophthalmic artery aneurysm: technical case report. Neurosurgery 58 (4 Suppl 2):ONS-E379, 2006

4. Dinc H, Kuzeyli K, Kosucu P, Sari A, Cekirge S: Retrieval of prolapsed coils during endovascular treatment of cerebral aneurysms. Neuroradiology 48:269-272, 2006

5. Ding D, Liu KC: Management strategies for intraprocedural coil migration during endovascular treatment of intracranial aneurysms. J Neurointerv Surg 6:428-431, 2014

6. Fiorella D, Albuquerque FC, Woo H, Rasmussen PA, Masaryk TJ, McDougall CG: Neuroform in-stent stenosis: incidence, natural history, and treatment strategies. Neurosurgery 59:34-42, 2006

7. Friedman JA, Nichols DA, Meyer FB, Pichelmann MA, McIver JI, Toussaint LG III, et al: Guglielmi detachable coil treatment of ruptured saccular cerebral aneurysms: retrospective review of a 10-year single-center experience. AJNR Am J Neuroradiol 24:526-533, 2003

8. Henkes H, Fischer S, Weber W, Miloslavski E, Felber S, Brew S, et al: Endovascular coil occlusion of 1811 intracranial aneurysms: early angiographic and clinical results. Neurosurgery 54:268-285, 2004

9. Heuer GG, Zaghloul KA, Roberts R, Stiefel MF, Storm 
PB: Successful microsurgical extraction of a migrated coil in a pediatric patient after failed endovascular closure of a Blalock-Taussig shunt. Case report. J Neurosurg 106 (2 Suppl):136-138, 2007

10. Hwang G, Jung C, Park SQ, Kang HS, Lee SH, Oh CW, et al: Thromboembolic complications of elective coil embolization of unruptured aneurysms: the effect of oral antiplatelet preparation on periprocedural thromboembolic complication. Neurosurgery 67:743-748, 2010

11. Kim YB, Lee KC, Lee JW, Huh SK, Yoon PH, Kim DI: Rescue microsurgery in coil herniation causing thromboembolic occlusion of parent artery. Acta Neurochir (Wien) 151:1609-1616, 2009

12. Kim YJ: Sole stenting technique for treatment of complex aneurysms. J Korean Neurosurg Soc 46:545-551, 2009

13. Kung DK, Abel TJ, Madhavan KH, Dalyai RT, Dlouhy BJ, Liu W, et al: Treatment of endovascular coil and stent migration using the Merci Retriever: report of three cases. Case Rep Med 2012:242101, 2012

14. Leslie-Mazwi TM, Heddier M, Nordmeyer H, Stauder M, Velasco A, Mosimann PJ, et al: Stent retriever use for retrieval of displaced microcoils: a consecutive case series. AJNR Am J Neuroradiol 34:1996-1999, 2013

15. Luo CB, Chang FC, Teng MM, Guo WY, Chang CY: Stent management of coil herniation in embolization of internal carotid aneurysms. AJNR Am J Neuroradiol 29:1951-1955, 2008

16. Mariak Z, Kochanowicz J, Kordecki K, Jadeszko M, Łysoń T, Lewko J: [Surgical evacuation of an embolization coil from the middle cerebral artery.] Neurol Neurochir Pol 38:533537, 2004 (Polish)

17. Molyneux A, Kerr R, Stratton I, Sandercock P, Clarke M, Shrimpton J, et al: International Subarachnoid Aneurysm Trial (ISAT) of neurosurgical clipping versus endovascular coiling in 2143 patients with ruptured intracranial aneurysms: a randomised trial. Lancet 360:1267-1274, 2002

18. Park HK, Horowitz M, Jungreis C, Genevro J, Koebbe C, Levy E, et al: Periprocedural morbidity and mortality associ- ated with endovascular treatment of intracranial aneurysms. AJNR Am J Neuroradiol 26:506-514, 2005

19. Pelz DM, Lownie SP, Fox AJ: Thromboembolic events associated with the treatment of cerebral aneurysms with Guglielmi detachable coils. AJNR Am J Neuroradiol 19:15411547, 1998

20. Raftopoulos C, Goffette P, Billa RF, Mathurin P: Transvascular coil hooking procedure to retrieve an unraveled Guglielmi detachable coil: technical note. Neurosurgery 50:912-915, 2002

21. Thornton J, Dovey Z, Alazzaz A, Misra M, Aletich VA, Debrun GM, et al: Surgery following endovascular coiling of intracranial aneurysms. Surg Neurol 54:352-360, 2000

22. Turek G, Kochanowicz J, Lewszuk A, Rutkowski R, Lysoń T, Kordecki K, et al: [Sonographic diagnosis and endovascular recanalization of the internal carotid artery in a patient with carotid thromboembolism following coiling of cerebral aneurysm.] Neurol Neurochir Pol 45:286-291, 2011 (Polish)

23. van der Schaaf IC, Brilstra EH, Buskens E, Rinkel GJ: Endovascular treatment of aneurysms in the cavernous sinus: a systematic review on balloon occlusion of the parent vessel and embolization with coils. Stroke 33:313-318, 2002

\section{Author Contributions}

Conception and design: Turek, Lewszuk, Lyson, Mariak. Drafting the article: Turek, Lewszuk, Mariak. Critically revising the article: Turek, Mariak. Approved the final version of the manuscript on behalf of all authors: Turek. Administrative/technical/material support: Turek, Kochanowicz, Lewszuk, Zielinska-Turek, Chwiesko, Mariak. Study supervision: Turek, Mariak.

\section{Correspondence}

Grzegorz Turek, Department of Neurosurgery, Medical University of Bialystok, Skłodowskiej-Curie 24a, 15-276 Bialystok, Poland.email: turekgrzegorz@vp.pl. 